\title{
A UNIFIED MODEL FOR KAKUTANI'S INTERVAL SPLITTING AND RÉNYI'S RANDOM PACKING
}

\author{
FUMIYASU KOMAKI* AND \\ YOSHIAKI ITOH, Graduate University for Advanced Studies
}

\begin{abstract}
One-dimensional random packing, known as the car-parking problem, was first analyzed by Rényi (1958). A stochastic version of Kakutani's (1975) interval splitting is another typical model on a one-dimensional interval. We consider a generalized car-parking problem which contains the above two models as special cases. In the generalized model, one can park a car of length $l$, if there is a space not less than 1 . We give the limiting packing density and the limiting distribution of the length of randomly selected gaps between cars. Our results bridge the two models of Rényi and Kakutani.
\end{abstract}

GENERALIZED RANDOM PACKING; PACKING DENSITY; DISTRIBUTION OF GAPS; ASYMPTOTIC BEHAVIOR

AMS 1991 SUBJECT CLASSIFICATION: PRIMARY 39B10

SECONDARY $60 \mathrm{~K} 99$

\section{Introduction}

We consider a generalization of Rényi's random packing problem. Cars of length, $l$ $(0 \leqq l \leqq 1)$ are allowed to park on the interval $[0, x]$ if there are spaces not less than 1 unit in length. Cars are sequentially parked, with the preferred locations of the front of each car being uniformly distributed on $[0, x]$. The car actually parks at this site if it fits within a gap of length $>1$. If not, it resamples its preferred location. The procedure continues until none of the gaps are $>1$. Here, we study this model and obtain closed formulas for the limiting packing densities and the limiting distribution functions of gaps. These bridge the two well-known formulas for the Rényi and Kakutani models.

Rényi's random packing is the special case $(l=1)$ of this problem. The case of $l=0$ is related to a stochastic version of Kakutani's interval splitting procedure and a stopping rule which was discussed by van Zwet (1978). For details of this relation, see van Zwet (1978).

\section{The limiting random packing density}

Let $M_{l}(x)$ be the expected number of cars allowed to park on the interval $[0, x]$. We have for $x \geqq 1-l$,

$$
M_{l}(x+l)=\frac{1}{x} \int_{0}^{x}\left\{M_{l}(y)+M_{l}(x-y)+1\right\} d y,
$$

with $M_{l}(x)=0$ for $0 \leqq x<1$. Put $w_{l}(s)=e^{s} \int_{0}^{\infty} M_{l}(x) \exp (-s x) d x$. We have

$$
\frac{d}{d s} w_{1}(s)=\left\{(1-l)-\frac{2 \exp (-l s)}{s}\right\} w_{l}(s)-\frac{1-l}{s}-\frac{1}{s^{2}} .
$$

Received 12 August 1991; revision received 18 December 1991.

Postal address for both authors: Department of Statistical Science, The Graduate University for Advanced Studies, and the Institute of Statistical Mathematics, 4-6-7, Minami Azabu, Minato-ku, Tokyo, 106, Japan.

* All correspondence should be addressed to this author. 


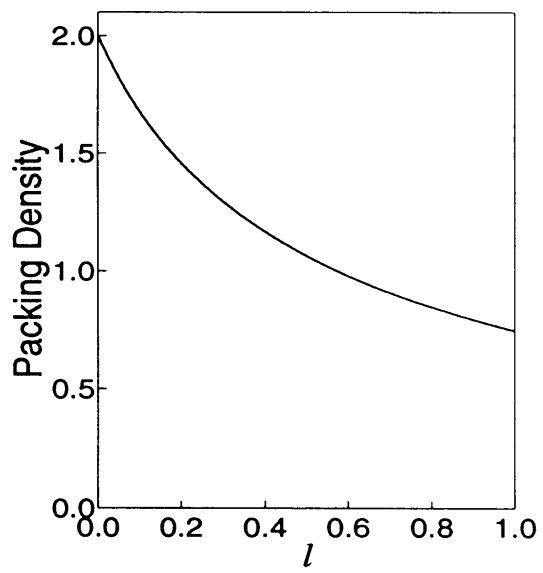

Figure 1. The limiting packing density $\lim _{x \rightarrow \infty} M_{l}(x) / x$ for $0 \leqq l \leqq 1$

The solution of (1), using the boundary condition $\lim _{s \rightarrow \infty} w_{l}(s)=0$, is

$$
w_{l}(s)=\frac{1}{s^{2}} \int_{s}^{\infty}\{1+(1-l) t\} \exp \{-(1-l)(t-s)\} \exp \left(-2 \int_{s}^{t} \frac{1-\exp (-l u)}{u} d u\right) d t .
$$

By the Tauberian theorem used in Rényi (1958),

$$
\lim _{x \rightarrow \infty} \frac{M_{l}(x)}{x}=\lim _{s \rightarrow 0} s^{2} w_{l}(s) \text {. }
$$

From this equality we obtain the following result.

Theorem 2.1. The limiting packing density of the cars of length $l(0 \leqq l \leqq 1)$ on the street of $x$ in length is given by

$$
\lim _{x \rightarrow \infty} \frac{M_{l}(x)}{x}=\int_{0}^{\infty}\{1+(1-l) t\} \exp \{-(1-l) t\} \exp \left(-2 \int_{0}^{t} \frac{1-\exp (-l u)}{u} d u\right) d t
$$

When $l=1$, the formula (3) coincides with the result obtained by Rényi (1958) in which $\lim _{x \rightarrow \infty} M_{1}(x) / x \simeq 0.748$. When $l=0, \lim _{x \rightarrow \infty} M_{0}(x) / x=2$ obtained from (3) corresponds to $M_{0}(x)=2 x-1(1 \leqq x)$ for a stochastic version of Kakutani's interval splitting problem. The limiting packing densities (3) are shown in Figure 1.

\section{The limiting distribution of a randomly chosen gap}

Let $m_{l, h}(x)$ be the sum of the expected number of cars and that of gaps which are bigger than $h$. We have for $x \geqq 1-l$,

$$
m_{l, h}(x+l)=\frac{1}{x} \int_{0}^{x}\left\{m_{l, h}(y)+m_{l, h}(x-y)+1\right\} d y,
$$

and the initial condition

$$
m_{l, h}(x)= \begin{cases}0, & 0 \leqq x<h \\ 1, & h \leqq x<1\end{cases}
$$


Put $\tilde{w}_{l, h}(s)=\exp (h s) \int_{1}^{\infty} m_{l, h}(x) \exp (-s x) d x$. We have

$\frac{d}{d s} \tilde{w}_{l, h}=\left\{(h-l)-\frac{2 \exp (-s l)}{s}\right\} \tilde{w}_{l, h}-\frac{2 \exp (h-l) s}{s} \int_{1-l}^{1} m_{l, h}(x) \exp (-s x) d x$

where

$$
-\frac{1-l}{s} \exp (-(1-h) s) m_{l, h}(1)-\frac{\exp (-(1-h) s)}{s^{2}},
$$

$$
m_{l, h}(1)=\left\{\begin{array}{cc}
1, & h+l \geqq 1 \\
3-\frac{2 h}{1-l}, & h+l<1
\end{array}\right.
$$

By an argument similar to the derivation of (2), we have

(4) $\lim _{x \rightarrow \infty} \frac{m_{l, h}(x)}{x}=\int_{0}^{\infty}\left[2 t \int_{1-l}^{1} m_{l, h}(x) \exp (-t x) d x\right.$

$$
\left.+\left\{(1-l) m_{l, h}(1) t+1\right\} \exp (-(1-l) t)\right] \exp \left(-2 \int_{0}^{t} \frac{1-\exp (-l u)}{u} d u\right) d t .
$$

By an argument analogous to the proof for Theorem 1 in Bankövi (1962),

$$
\lim _{x \rightarrow \infty} \operatorname{Pr}\left(I_{x, l} \leqq h\right)=2-\frac{\lim _{x \rightarrow \infty} m_{l, h}(x) / x}{\lim _{x \rightarrow \infty} m_{l, 1}(x) / x} .
$$

Here, $I_{x, l}$ is the length of the randomly chosen gap.

We can get the following theorem from (4) and (5).

Theorem 3.1. Let $I_{x, l}$ be the length of a randomly chosen gap. The limiting distribution function of $I_{x, l}$ as $x \rightarrow \infty$ is given by

$\lim _{x \rightarrow \infty} \operatorname{Pr}\left(I_{x, l} \leqq h\right)$

$(6)=\left\{\begin{array}{c}1-2 \frac{\int_{0}^{\infty}(-\exp (-t)+\exp (-h t)) \exp \left(-2 \int_{0}^{t} \frac{1-\exp (-l u)}{u} d u\right) d t}{\int_{0}^{\infty}\{(1-l) t+1\} \exp (-(1-l) t) \exp \left(-2 \int_{0}^{t} \frac{1-\exp (-l u)}{u} d u\right) d t}, \quad h+l \geqq 1 \\ \int_{0}^{\infty}\{-\exp (-t)+\exp (-(1-l) t) \\ +2 \frac{(1-l-h) t \exp (-(1-l) t)\} \exp \left(-2 \int_{0}^{t} \frac{1-\exp (-l u)}{u} d u\right) d t}{\int_{0}^{\infty}\{(1-l) t+1\} \exp (-(1-l) t) \exp \left(-2 \int_{0}^{t} \frac{1-\exp (-l u)}{u} d u\right) d t}, \\ 1-2+l<1 .\end{array}\right.$

The limiting density functions of $I_{x, l}$ for several values of $l$ are illustrated in Figure 2 . When $l=1$, the formula (6) coincides with the result by Bankövi (1962). When $l=0$, (6) gives $\lim _{x \rightarrow \infty} \operatorname{Pr}\left(I_{x, l} \leqq h\right)=h$. 


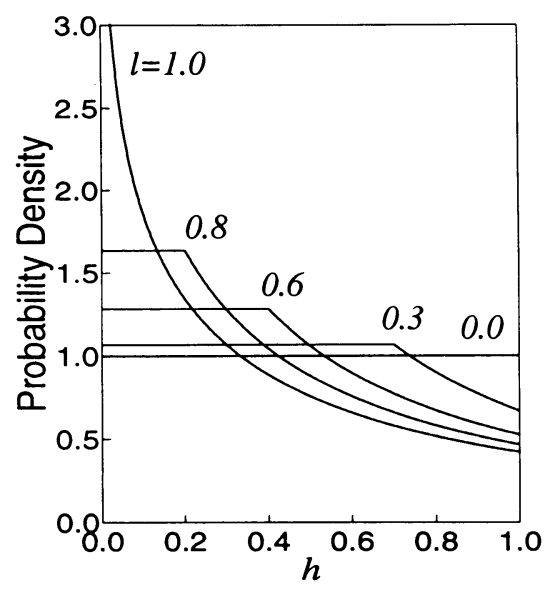

Figure 2. The limiting density functions of a randomly chosen gap between cars for several values of $l(l=0.0,0.3,0.6,0.8,1.0)$

\section{References}

BANKövi, G. (1962) On gaps generated by a random space filling procedure. Publ. Math. Inst. Hungar. Acad. Sci. 7, 395-407.

KAKUTANI, S. (1975) A problem of equidistribution on the unit interval $[0,1]$. Lecture Notes in Mathematics 541, 369-376.

RÉNYI, A. (1958) On a one-dimensional problem concerning random space-filling problem. Publ. Math. Inst. Hungar. Acad. Sci. 3, 109-127.

VAN ZWET, W. R. (1978) A proof of Kakutani's conjecture on random subdivision of longest intervals. Ann. Prob. 6, 133-137. 